\title{
Electrophilicities and protein covalent binding of demethylation
} metabolites of colchicine

\author{
Xiucai Guo, ${ }^{\dagger}$ Dongju Lin, ${ }^{\dagger}$ Weiwei Li, ${ }^{\S}$ Kai Wang, ${ }^{\dagger}$ Ying Peng, ${ }^{\dagger} *$ and Jiang Zheng ${ }^{\S \mathscr{I} *}$ \\ ${ }^{\dagger}$ School of Pharmacy, ${ }^{\S}$ Key Laboratory of Structure-Based Drug Design \& Discovery of Ministry of \\ Education, Shenyang Pharmaceutical University, Shenyang, Liaoning, 110016, P. R. China \\ ITCenter for Developmental Therapeutics, Seattle Children's Research Institute, Division of \\ Gastroenterology and Hepatology, Department of Pediatrics, University of Washington School of \\ Medicine, Seattle, WA 98101
}

\section{Supporting Information:}

Figure S1. Extracted ion $(\mathrm{m} / z 547 \rightarrow 418)$ chromatograms obtained from LC-MS/MS analysis of the incubations of human liver microsomes containing colchicine and NAC in the absence (A) and presence (B) of NADPH.

Figure S2. Extracted ion $(\mathrm{m} / z, 505 \rightarrow 418)$ chromatograms obtained from LC-MS/MS analysis of proteolytic digestion of human liver microsomes after exposure to colchicine in the absence (A) or presence (B) of NADPH. 

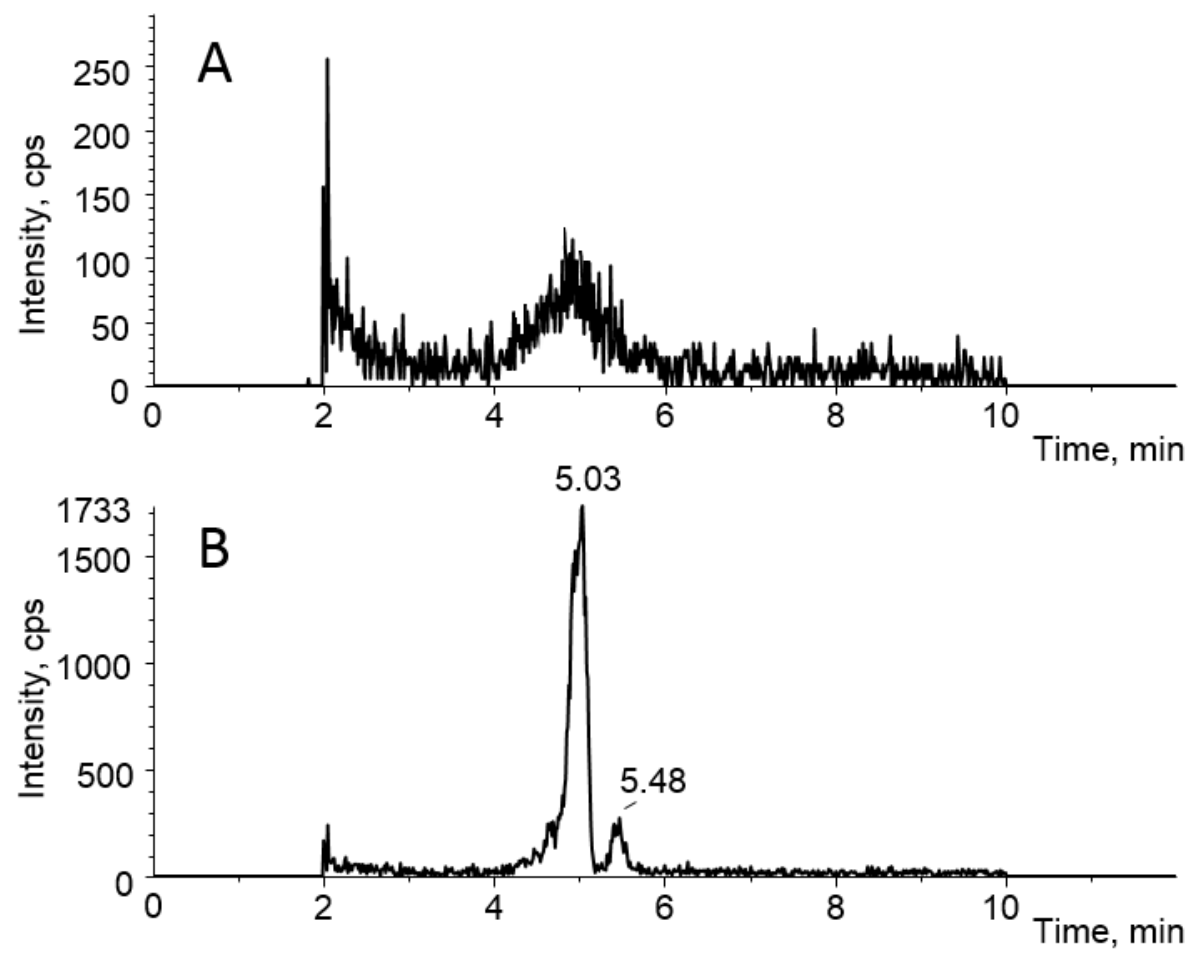

Figure S1. Extracted ion $(\mathrm{m} / \mathrm{z}, 547 \rightarrow 418)$ chromatograms obtained from LC-MS/MS analysis of the incubations of human liver microsomes containing colchicine and NAC in the absence (A) and presence (B) of NADPH. 

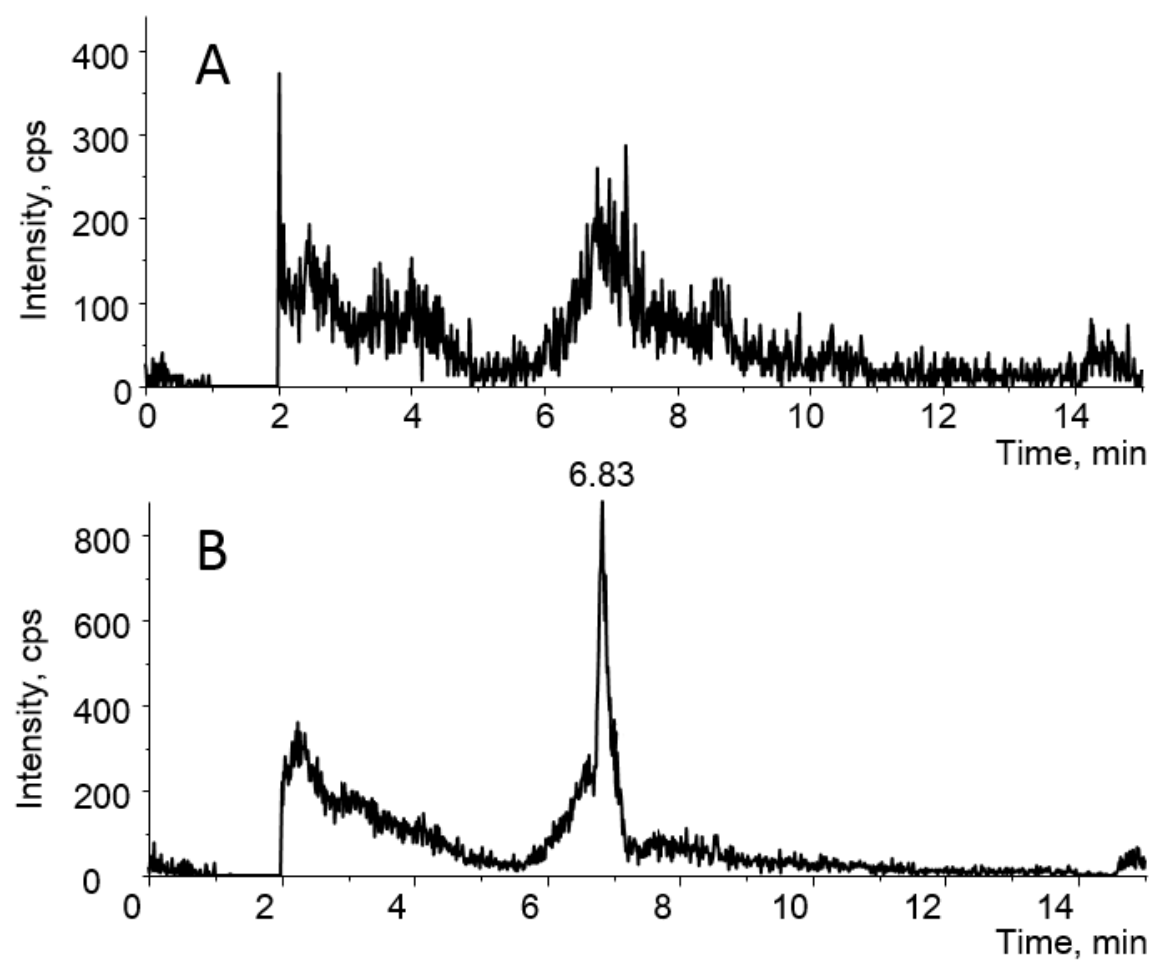

Figure S2. Extracted ion $(\mathrm{m} / \mathrm{z} 505 \rightarrow 418)$ chromatograms obtained from LC-MS/MS analysis of proteolytic digestion of human liver microsomes after exposure to colchicine in the absence (A) or presence (B) of NADPH. 